\title{
Exploration of task-based scheduling for convolutional neural networks accelerators under memory constraints
}

DOI:

10.1145/3310273.3323162

\section{Document Version}

Accepted author manuscript

Link to publication record in Manchester Research Explorer

\section{Citation for published version (APA):}

Rodrigues, C. F., Riley, G., \& Luján, M. (2019). Exploration of task-based scheduling for convolutional neural networks accelerators under memory constraints. In 5th Workshop on design of Low Power EMbedded Systems at Computing Frontiers 2019 (pp. 366-372) https://doi.org/10.1145/3310273.3323162

\section{Published in:}

5th Workshop on design of Low Power EMbedded Systems at Computing Frontiers 2019

\section{Citing this paper}

Please note that where the full-text provided on Manchester Research Explorer is the Author Accepted Manuscript or Proof version this may differ from the final Published version. If citing, it is advised that you check and use the publisher's definitive version.

\section{General rights}

Copyright and moral rights for the publications made accessible in the Research Explorer are retained by the authors and/or other copyright owners and it is a condition of accessing publications that users recognise and abide by the legal requirements associated with these rights.

\section{Takedown policy}

If you believe that this document breaches copyright please refer to the University of Manchester's Takedown Procedures [http://man.ac.uk/04Y6Bo] or contact uml.scholarlycommunications@manchester.ac.uk providing relevant details, so we can investigate your claim.

\section{OPEN ACCESS}




\section{Exploration of Task-based Scheduling for Convolutional Neural Networks Accelerators under Memory Constraints}

\author{
Crefeda Faviola Rodrigues \\ crefeda.rodrigues@manchester.ac.uk \\ The University of Manchester
}

\author{
Graham Riley \\ graham.riley@manchester.ac.uk \\ The University of Manchester
}

\author{
Mikel Luján \\ mikel.lujan@manchester.ac.uk \\ The University of Manchester
}

\begin{abstract}
Development of application specific accelerators for deep convolutional neural networks (ConvNets) have mainly focussed on accelerating the computationally intensive layers, that is the convolutional layers, to improve performance and energy efficiency. Traditional approaches in this space have relied on handcrafted dataflow implementations to leverage the fine-grained parallelism and datalocality properties within these layers. However, ConvNets layers also have an untapped potential from cross-layer data locality.

In our work, we explore a novel approach in the context of deep neural networks accelerators by modelling the computation as a task-dependency directed acyclic graph and proposing a memoryaware heuristic based on Heterogeneous Earliest Finish Time (HEFT) for task-graph scheduling on shared memory systems.

Our results show the benefits of task graphs in terms of better memory use (23.4\% less) over conventional layer-by-layer processing in a simulated environment with the first three layers of LeNet-5. Certain task-graphs trade-off makespan (10\% increase) for memory use (20\% decrease). Finally, our exploration of graphs with different slicing configurations for the pooling layer while using memory-aware HEFT versus the original HEFT reveals that regular shaped tiles across layers offers better makespan and memory use than tiles with large dimensions along one axis.
\end{abstract}

\section{CCS CONCEPTS}

- General and reference $\rightarrow$ Design; $\bullet$ Theory of computation $\rightarrow$ Parallel computing models; • Computing methodologies $\rightarrow$ Simulation evaluation; Object recognition.

\section{KEYWORDS}

Convolutional neural networks, scheduling, task-based parallelism, accelerator systems

\section{INTRODUCTION}

Convolutional neural network (hereafter referred to as ConvNets) models are major components in predictive tasks for computer vision and language processing problems [5]. There is a growing demand to enable ConvNet inferences on edge-devices that encompass the mobile and embedded market. This demand is motivated by the need to enable user privacy, mitigate the need for constant internet connectivity, improve the response time and reduce the burden on datacenters [10]. However, these devices are characterised by limited resources, for example, energy [2]. Moreover, the landscape of deep neural network architecture is ever-changing with new model designs that incorporate multiple filter choices $(1 \times 1,3 \times 3$ or $5 \times 5)[7,13]$, multiple layers (5 100's layers) $[5,9]$ and multiple topologies ${ }^{1}$ are being proposed. Hence, this growth in computation and memory requirements has made traditional computing platforms with CPUs and GPUs impractical for resource-constrained scenarios [10].

This has led to the search for a custom deep neural network accelerator designs that can be optimized for energy use [3, 4, 8, 12]. The memory requirements in executing ConvNets is an important concern as a large number of intermediate feature maps are generated between layer processing leading to overheads in energy use. Typical designs try to exploit data locality properties present in each layer. These designs are often based on spatial architectures consisting of multiple homogeneous cores (processing elements or PEs) and a memory hierarchy with multiple levels of cache memory connected to main memory. They rely on specialized techniques to map the computations within a layer onto the PEs with the goal of maximizing data reuse and thus reducing access to the main memory. This is referred to as a dataflow mapping [3]. This approach can be understood as two sub-problems of tiling and mapping. However, this classification is not a strict division and these steps are typically tightly coupled [4]. Tiling (also referred to as blocking in [16]) breaks the computation into smaller operations (referred in our work as the granularity for a task) [2]. Mapping refers to how these tasks are mapped onto resources such as, single array of PEs, as described in Eyeriss [3] or mapping across multiple arrays of PEs, as described in Tetris [4] (also referred to here as partitioning) and the order in which these tasks are executed on a resource [2-4].

However, unlike these previous approaches there is a lack of studies or tools to explore cross-layer dependencies (a notable exception is [1]). Moreover, such techniques can have significant implications on key accelerator architectural features, for example, number of PEs and shared memory size. In this work, we describe a novel way to express the dataflow in a ConvNet using task dependency graphs such that we can explore the data locality both within and across layers. We provide tunable knobs to study the impact of such a representation on the design of accelerator system, such as the number of cores, size of shared memory or the complexity of the DMA engine. Our contributions are the following:

- We model the computation of a convolutional neural network (Section 2) as a task-dependency Directed Acyclic Graph (DAG) (Section 4.1), where a task is a unit of computation that receives input and produces an output, and the edges represent the dataflow dependencies. Our approach explores the ConvNet as a classical task-parallel mapping problem.

- We extend an existing static scheduling strategy (Section 4.2) to incorporate shared memory constraints such as those found in current ConvNet accelerator designs.

${ }^{1}$ wide models [7] versus stacked models [13]. 
- We explore various tiling and mapping configurations of task graphs under memory-constraint aware HEFT to show the effects of our modification in restricted memory scenarios (Section 5) over the original HEFT.

\section{CONVNET MODEL STRUCTURE}

This section gives a brief overview on convolutional neural networks (ConvNets) at the algorithmic level. A ConvNet is one type of neural network model that consists of a hierarchical arrangement of layers. To a machine learning practitioner, this layeredrepresentation abstracts the type of computation performed at each step in the hierarchy. For example, typical layers found in a ConvNet include convolutional (conv), pooling, normalization, non-linear and fully-connected (fc) layers [11].

Shown in Figure 1, are two layers in a typical ConvNet model, for example, as seen in the first two layers of LeNet-5 [11]. Most of the layers in a ConvNet, receives a 3D volume of data (represented by the dimensions $X, Y$ and $Z$ ) and outputs a 3D volume of data to the next layer. For example, a convolutional layer receives its input referred to as input feature map, of dimension $\left(I_{x} \times I_{y} \times I_{z}\right)$, and performs a convolution operation using a filter $\left(K_{x} \times K_{y} \times I_{z}\right)$ to produce output feature maps $\left(O_{x} \times O_{y} \times O_{z}\right)$. Note, there are $O_{z}$ distinct conv filters that produces the output feature maps. These output feature maps in turn become input feature maps to the subsequent layer, which in this case, is the pooling layer $\left(P_{x} \times P_{y} \times\right.$ $O_{z}$ ). Each filter application looks at a specific region of the input, this is referred to as the receptive field of the filter on the input. The dimension of this region is specified by the conv filter dimensions. Each filter is then applied at a specific stride of the input. The value of the stride dictates the amount by which the receptive field moves across the input resulting in opportunities for data-reuse, which in this case refers to convolutional ${ }^{2}$.

Similarly, a pooling layer receives this input and performs a reduction operation, either Max pooling or Average pooling to produces output feature maps for the next layer [11].

\section{RELATED WORK}

This sections describes the current accelerator architecture designs and the corresponding dataflow implementation chosen for implementing ConvNets.

${ }^{2}$ Please refer to [3] for a full taxonomy on types of reuse present within a conv layer.

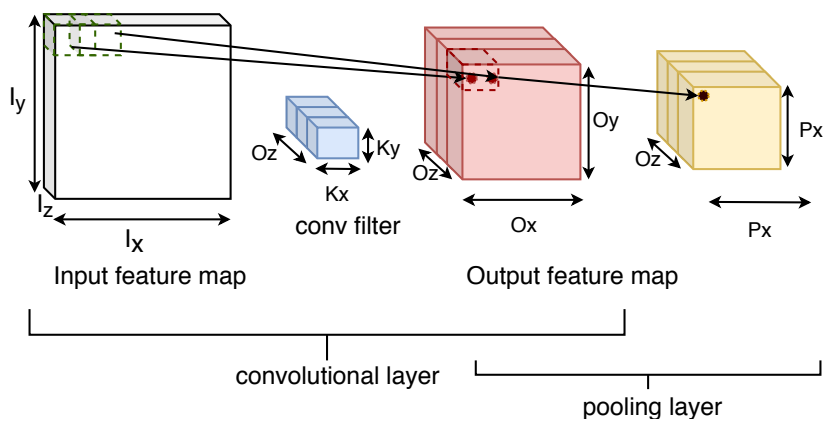

Figure 1: A simple two layer ConvNet model

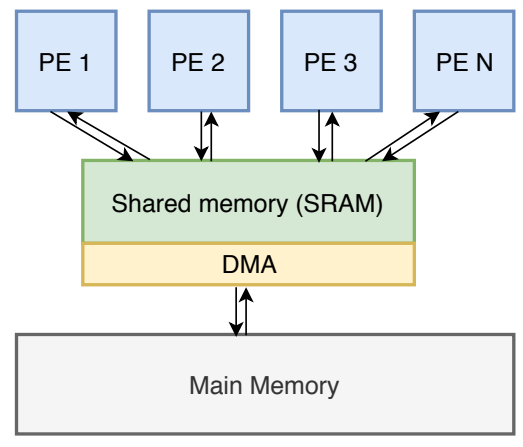

Figure 2: A model of the system architecture

Eyeriss-version1 [3] is an example of a single 2D array architecture consisting of a 2D-mesh of processing elements, each of them equipped to perform a multiply-and-accumulate (MAC) and an addition. It consists of a four-level memory hierarchy (a register file local to each PE, inter-PE, a global buffer shared among all PEs and DRAM), each with a different energy cost for access. The main design goal was to map the computation within a conv layer on the 2D array of PEs by exploiting data reuse to reduce DRAM access and maximize the use of the PEs. To enable this, they use a row stationary dataflow mapping technique, described in [3]. Once the results of processing a single layer is completed, the output feature maps are then transferred to main memory [12]. However, we express the problem as a graph of task dependencies to explore the possibility of data that transcends layer boundaries.

To support larger ConvNets by scaling the number of PEs and increasing buffer size for the Eyeriss-version1 was considered impractical in Tetris [4]. This is due to the increase bandwidth requirements resulting in an increase in static power consumption of accessing memory. They aim to scale this design by having multiple arrays of PEs arranged into vaults coupled within a 3D memory stack. Additionally, they rely on two hand-crafted techniques a) an ordering scheme to bypass storing either input or output feature maps into global buffer and directly streaming them into the register files b) a partitioning scheme to split the computation into fixed-sized tiles across multiple vaults. We wish to target a more general version of the architecture adopted in Tetris consisting of multiple PE arrays connected to a shared memory.

Other studies such as [2], have looked for the optimum tiling strategy for each layer that minimize accesses to main memory. However, the mapping adopted was a simple sequential access of tiles. In our work, we aim to jointly explore the two-sub problems of tiling and mapping problem for a baseline accelerator system (see section 4 for further details), by framing it as a task-graph scheduling problem. Unlike previous approaches, our mapping strategy looks at the entire DAG with tasks across multiple layers and prioritizes the tasks based on some cost metric associated to each task. It then uses a heuristic-based approach to search for a good schedule for these tasks based on time while managing memory constraints on a simulated shared memory system. 


\section{METHODOLOGY}

To facilitate this study of the tiling and mapping in the design space of ConvNet implementation and hardware choices, we consider a base system shown in Figure 2. This design adheres to typical accelerator designs found in the literature, consisting of a number of PEs connected to a small and fast shared memory $[3,12,14]$. The computational capability of a PE can either be homogeneous, where a task has a similar processing time on all cores, or heterogeneous, where a task has a variable processing time on all cores. This is equivalent to having a bunch of simple PE arrays, found in accelerators like Eyeriss [3] for the former, or a big.LITTLE architecture which uses combination of simple power-efficient cores (LITTLE) or powerful but power-hungry cores (big) ${ }^{3}$ for the latter. Each PE has a uniform memory access to the shared memory. Memory accesses to-and-fro from the main memory is controlled by the Direct Memory Access (DMA) engine.

Our methodology, as shown in Figure 3, consists of two main steps a) Directed Acyclic Graph (DAG) generator for a ConvNet that models the computation in a layer into tasks and generates a graph with dependency relations between tasks across layers b) a static scheduling algorithm that takes as input a given DAG specification and computes a schedule on the base system with a specific configuration evaluated on the makespan (a metric of time) and memory use.

The DAG generator receives as input the ConvNet layer dimensions, as described in Section 2 and information on how to form tasks (described in Section 4.1 as slicing parameters and data types). The output of the DAG generator is a task graph for the ConvNet with information for the processing time for each task, memory requirements and the dependencies between tasks.

Our scheduler configurable parameters can be used to specify the configuration of the base system. Shared memory size is the amount of shared SRAM memory for all the PEs that can be used to store data (described in Section 4.1, as tiles) from both the input feature maps and output feature maps. In this work, we assume the storage of filter weights to be free, for example, loaded into register files. Tile volume represents a portion of the shared memory reserved for the input data read by a task, or a portion of the shared memory for a task to write to. Finally, the number of PEs dictates the number of tasks that can be simultaneously processed.

\subsection{Directed Acyclic graph generator}

The Directed Acyclic Graph (DAG) generator models the computation in a ConvNet into a set of inter-dependent tasks. We define a task as an independent operation that can be performed on an input or multiple inputs to produce an output, as shown in Figure 4. The input and outputs are defined for a region of the original input and output feature map space and this region (shown by the dotted box) can fill an allocated space in shared memory called a tile. The amount of space allocated for a tile is referred to as the tile volume. We specify the slice of data (in the the $\mathrm{X}, \mathrm{Y}$ and $\mathrm{Z}$ dimension according to the $3 \mathrm{D}$ representation of the data used in ConvNet) to try and completely fill this tile volume, using the slicing parameters Note that a conv filter can be applied at the boundary of input tiles. Therefore, the task can receive partial data across tile boundaries.

\footnotetext{
${ }^{3}$ https://developer.arm.com/technologies/big-little
}

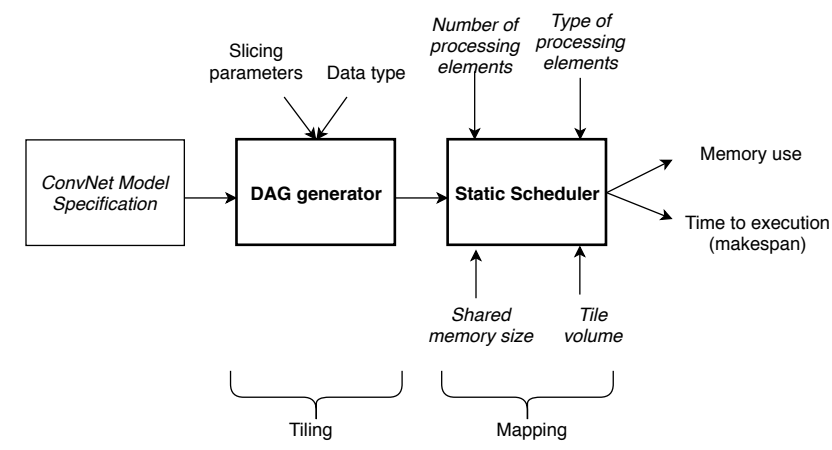

Figure 3: Tiling and mapping methodology

In this example, we also see that input tile 2 does not cover the input feature map space completely. We define this as the tile occupancy as the amount of the input or output feature map space that can fill a tile volume. The tile occupancy is also affected by the data type (in bytes) of the input and output feature map. Data represented in $4 B$ (32bits) will have lesser data elements than that of data in $1 B$. Similarly, a task can write a region of the output feature map space that may or may not completely fill an output tile, thus affecting tile occupancy.

These different choices in the slicing parameters will result in different DAGs with different number of tasks and different dependency relations. Each DAG will then have different memory requirements for tasks, that is, the number of tiles that can fit in shared memory at any given time during the scheduling step and opens up the opportunity to study data reuse patterns when dealing with cross-layer data locality. Similarly, we can extract independent tasks at other layers, such as pooling, because these layers are also a collection of operations applied to a specific region of the previous layer input.

We can now describe a DAG by defining the four components $\mathrm{N}, \mathrm{E}, \mathrm{W}$ and $\mathrm{C} . \mathrm{N}$ represents a set of tasks, $\mathrm{E}$ is the set of edges between tasks that enforces the dependency relations. $\mathrm{W}$ is a set of weights for each task that represents the computation cost of each

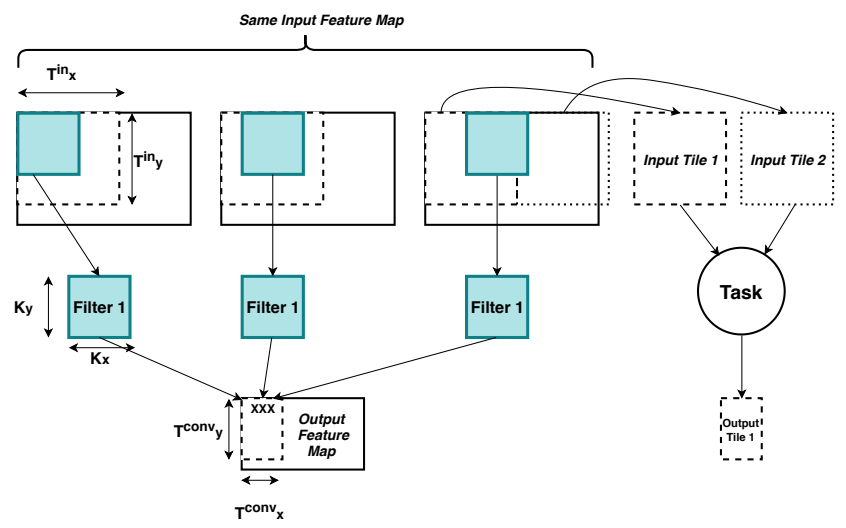

Figure 4: Tile and Task formation for the 2D case 
task in time and $\mathrm{C}$ typically models the set of communication costs between tasks. We are dealing with a shared memory system, and as described previously, each task has a set of input tiles it depends on and produces an output tile. Therefore, this communication cost has to be modified during the scheduling phase, where tasks pay this cost only if its dependent tiles are not present in memory.

The slicing of feature maps to fill tiles is now described for two layers: the input layer and the conv layer.

4.1.1 Slicing the input layer: The input layer is typically an image in a ConvNet. This represents an external input to the ConvNet model, and in a typical DAG representation they are represented as pseudo tasks with zero computational cost. The slicing can be done in $\mathrm{X}, \mathrm{Y}$ and $\mathrm{Z}$ with no constraints. For example, the input feature map of dimensions $\left(I_{x} \times I_{y}\right)$ can be split in $\mathrm{X}$ and $\mathrm{Y}$ into slices of dimensions $\left(T_{x}^{i n} \times T_{y}^{i n}\right)$ shown in Figure 4 . A similar procedure can be applied in the $\mathrm{Z}$ dimension.

4.1.2 Slicing other layers: When slicing other layers, we have to keep in mind that we want a task to ideally fill an output tile. We also assume that a task can write to a single output tile so that writes can be done independently to different locations in the shared memory. Therefore, the granularity of a task is dictated by the region of the output feature map that can be produced to fill an output tile. As shown in Figure 4, there are three applications of the same conv filter on the input feature map along X dimension. These three filter applications results in three output elements that fill an output tile. This is represented as $\left(T_{x}^{c o n v} \times T_{y}^{c o n v}\right)$. Along $\mathrm{Z}$ in Figure 1, each distinct filter generates a unique feature map (represented as $O_{z}$ ), we can therefore extract independent slices along this dimension.

4.1.3 Building the dependency relation: We know the dependencies of the conv output point to its input tiles based on whether the filter window resides within an input tile or across multiple input tiles. The output tiles of the current layer in-turn serves as input tiles to the next layer tasks for processing. This dependency information can be used to build a task-task dependency relation given by the edges $E$ of the DAG.

4.1.4 Assignment of costs to a task: This step generates the information that is required by the scheduler to compute the makespan and memory use. Given that the shapes of the output feature maps for a given ConvNet is fixed, we can pre-compute the computational cost of a task based on its tile occupancy and the number of operations to produce each output point in the occupied tile, given in Equation 1 and the cost of an operation. For example, each conv output point has $\left(K_{x} \times K_{y} \times I_{z}\right)$ number of multiply-accumulate (MAC) operations. We assume each MAC takes 1 unit of time to compute (opCost $=1$ unit).

$$
\operatorname{compcost}(\operatorname{conv})=(\text { tileOccupancy }) \times\left(K_{x} \times K_{y} \times I_{z}\right) \times \text { opCost }
$$

The next cost that is computed is the communication cost, given in Equation 2. This is based on the number of bytes that needs to be loaded from main memory into the shared memory and the relative cost of loading a byte of data to the cost of an operation. The number of bytes that has to be loaded from main memory for a task is a function of the number of dependent input tile $N$ and plus one for the output tile, and the tile occupancy of each tile.

$$
\operatorname{commcost}(\operatorname{conv})=\left(\sum_{n}^{N+1}(\text { tileOccupancy }) \times(\text { relativeCost })\right.
$$

This section gave an overview of how a DAG can be generated for a input-conv layer boundary. A similar approach can be carried out for other layers. It provides information related to the task dependency relations across layers, a computation cost for tasks in a layer and communication costs that represents the memory requirements per task.

\subsection{Scheduling}

A scheduling system typically allocates each task of the graph to a suitable resource (for example, a processor) on a target computing platform. The goal of the scheduler is to schedule or map all the tasks to resources based on some criteria, for example, makespan (time to completion from the start time of the first task to the finish time of the final task). If prior information about the task is available, for example, the computation time and memory requirements for each task, the scheduler can use this information to generate a better allocation [15]. HEFT is a widely studied heuristic-based scheduling strategy for task scheduling problems on heterogeneous systems that can generate a close to optimal static schedule in time for a task graph. It does this in two phases: a task ordering phase for computing the priority for each task and a processor selection phase for allocating tasks to a processor based on a heuristic that optimizes for time. It is important to highlight that the schedule is generated for a simulated target environment.

The original heuristic in HEFT does not optimize for memory, and considers the availability of unlimited amount of memory to support parallel execution of tasks. Moreover, the only limit to the parallel execution of tasks is the number of processors on the system. Previous work [6], introduced a memory-aware heuristic for HEFT on a system consisting of distributed memory. However, we extend it to enforce memory use constraints in a shared memory system while still using the capabilities of the original HEFT to derive a schedule that minimizes makespan. We modify the task ordering heuristic and incorporate a memory manager in the processor selection phase to generate schedules for a shared memory system. In our work, the memory requirement or memory use refers to the maximum number of tiles simultaneously in shared memory. Our work can support future opportunities to search for heuristics that can optimize for both memory use and makespan.

4.2.1 Task ordering phase: In this section, we first introduce the HEFT ordering phase and highlight our modifications for shared memory constraints. This phase calculates the priority (also referred to as rank) of each task to place tasks on the critical path with highest priority first. For example, the priority can be used to place a task with high computational time and memory requirements first such that it has the best chance of being placed on a faster processor if available. In our work, the priority is determined using the computational and communication costs that was computed in the DAG generator step (see Equation 1 and Equation 2). The computational time is averaged over all processors $P$ and is given by $W_{\text {comp }}(i)=\sum_{k}^{P} \operatorname{compcost}(i)^{k} /(\alpha \times P)$. In our case, these are all 
homogeneous processors $(\alpha=1)$ but can be weighted to account for heterogeneous processors.

The communication cost in the original HEFT refers to the amount of data to be transferred from the parent task to the child task if they are not on the same processor. We modify this communication cost to reflect the maximum memory requirements in terms of dependent input tiles and output tile of a task. This cost is then modified during the processor selection phase to be paid only if the dependent input tiles are not in shared memory. The priority is computed recursively for the current task and its children, and is given by Equation 3.

$\operatorname{rank}(i)=W_{\text {comp }}(i)+\operatorname{commcost}($ parents $(i))+\max _{j \in \operatorname{children}(i)}\{\operatorname{rank}(j)\}$

The tasks are then sorted based on the highest rank. Therefore, our rank formulation prioritizes the tasks with higher computational time and dependencies first.

4.2.2 Processor selection phase: In this section, we first introduce the HEFT processor selection phase before describing our modifications for shared memory constraints. HEFT selects each tasks from a sorted list of task to be placed on a processor. The placement is based on the following criteria : a) precedence constraint: completion of all parent tasks before the execution of the current tasks b) processor availability. We extend this to include two more criteria: c) availability of memory resources to store input tiles from parent tasks and the output tile from current task d) all input tiles to be present memory before execution begins using a tileload cost.

A schedule is obtained when all tasks have been allocated to a resource while satisfying a performance criteria on makespan and memory use. In a schedule, each task $i$ has an estimated start time $\operatorname{EST}(i, p)$ and an estimated finish time $\operatorname{EFT}(i, p)$ on a given processor $p$. The $E S T(i)$ of task $i$ is a function of the possible start times derived from the precedence constraint, memory availability and tile loading costs and the availability of a free processor. The precedence constraints ensures all preceding tasks must be scheduled, and is given by:

$$
\text { precedence_EST }(i)=\max _{n \in \text { parents }(i)}\{A F T(n)\}
$$

Here, AFT refers to the actual finish time of the preceding task $n$. The availability of processor checks for the AFT of the last task allocated to it, and is given by:

$$
\text { processor_EST }(i)=\max _{n_{p} \in \text { allocation }(p)}\left\{\operatorname{AFT}\left(n_{p}\right)\right\}
$$

We select a desired time to start a task as per the original HEFT, which is given by:

$$
\text { desired_EST }(i)=\max \{\text { precedence_EST }(i), \text { processor_EST }(i)\}
$$

Similar to previous approaches [6], we maintain a function mem_state $(t)$ to store a tuple list of time and memory use. However, our tuple list is maintained based on the tiles loaded in shared memory at any given time $t$ and a separate list valid( $n)$ to store the start and end times of tile $n$ that may have been loaded thus far. Once we select a desired_EST $(i)$, we use the mem_state $(t)$ to check which tiles are already present in memory for the duration of the current task $($ duration $=\operatorname{compcost}($ task $))$. If tiles are required to be loaded

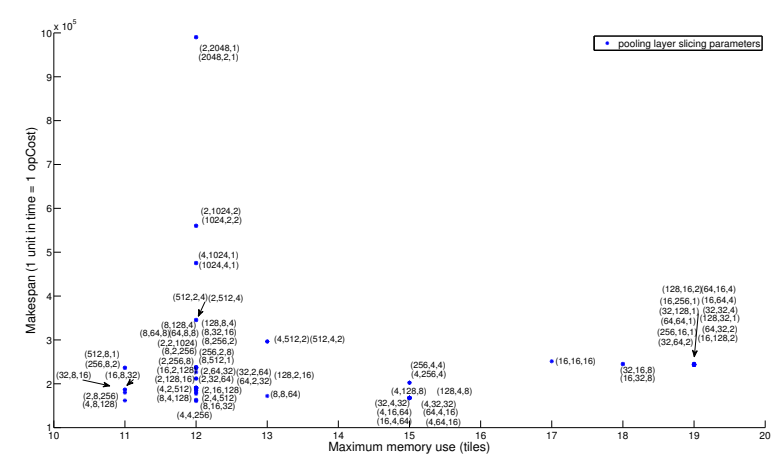

Figure 5: Pooling slicing parameters for a fixed input $8 \times 8 \times 64$ and conv $8 \times 8 \times 64$ - original HEFT - no memory restriction

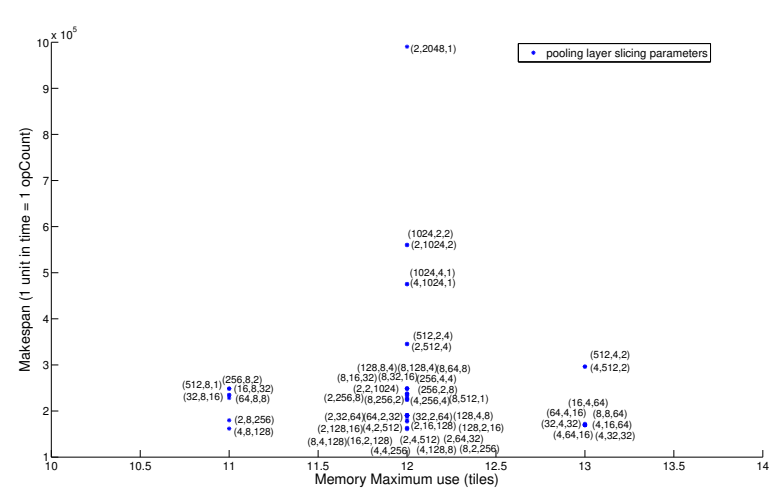

Figure 6: Pooling slicing parameters for a fixed input $8 \times 8 \times 64$ and conv $8 \times 8 \times 64$ - modified HEFT- memory restriction

from main memory we pay the load cost for each tile (Note: this is our modification to the communication cost described in task ordering phase). A tile load cost is a function of the number of tiles not loaded in shared memory $\left(N_{\text {subset }}\right)$, and the amount of data present in such tiles, see Equation 7 . The motivation for this is that the DMA engine could specialized to load only valid data in a tile into shared memory or be specialized to use compression techniques ${ }^{4}$. We assume a task can start only when all its dependent tiles are loaded in memory.

$$
\text { tileLoadCost }(i)=\sum_{n \in \text { parents }(i)}^{N_{\text {subset }}} \text { tileOccupancy } y_{n} \times(\text { relativeCost })
$$

The new desired time after tile loading is new_desired_EST $(i)=$ desired_EST $(i)+$ tileLoadCost $(i)$. We now check if there is sufficient memory for all the tiles already present in memory plus unloaded dependent tiles for the current task. If memory is not available, we differ the placement of the current task until memory is available. 


\section{RESULTS}

In this section, we show the effects of our HEFT modifications (with shared memory) with a baseline HEFT (with no shared memory) for different task graphs. These task graphs are generated using the first three layers for an example ConvNet known as LeNet-5 [11]. The LeNet- 5 model structure consists input $(32 \times 32 \times 1)$, conv layer with filter dimensions $\left(5 \times 5 \times 16^{5}\right)$ and stride 1 and pooling with a $2 \times 2$ window and stride 2 .

We provide this definition of LeNet-5 to the DAG generator to generate all possible DAGs with a fixed slice for the input layer $(8 \times 8 \times 64)$ and conv layer $(8 \times 8 \times 64)$ and all possible pooling layer slicing parameters. Our example base accelerator design consists of 3 processors ( $a, b$ and $c$ ) with equal computational capability $\alpha=1$ and initially an unlimited amount of shared memory. This corresponds to the original HEFT version. We later fix the size of the shared memory and explore its impact on each DAG. The tile volume is fixed to $4 \mathrm{~KB}$ (a typical page size) and data type is $1 \mathrm{~B}$.

Figure 5 shows a collection of DAGs where each point represents a single DAG that has a unique pooling layer slicing parameter choice. We label each data point with only the pooling layer slicing parameters. The $\mathrm{X}$-axis represents the maximum memory use, which is the maximum number of tiles simultaneously in shared memory at any given time and $\mathrm{Y}$ axis represents the makespan of the DAG. We observe that slicing parameters that have a large axis dimension perform worse, in both makespan and memory use compared to slicing parameters that are more regular shaped ${ }^{6}$. For example DAG $(2,2048,1)$ performs worse than DAG $(4,8,128)$ because the latter has a similar shaped slicing parameter to the preceding conv layer leading to a DAG with lesser dependencies in the pooling layer.

We now impose some restriction on shared memory (to hold 13 tiles at any given instant) to all the DAG configurations from the previous step as shown Figure 6. We observe that DAGs with memory requirements below the restriction do not change positions in terms of makespan and memory use while certain DAGs, with memory requirements above the restriction are forced to meet the memory requirement at the expense of makespan. For example, all DAGs with different permutations of pooling configuration parameters of $(4,16$ and 64$)$ that were originally clustered around memory use of 15 are now forced to execute with a memory use of 13 at the expense of a slight increase in makespan. Additionally, $23 \%$ DAGs have to be aborted as they can not fulfil the minimum memory requirement for a task at any given instant. For example, the DAG with pooling configuration $(16 \times 16 \times 16)$ with memory use of 17 tiles does not satisfy this requirement.

We also take an example DAG with pooling configuration $(4 \times$ $256 \times 4$ ) from Figure 5 and Figure 6 and illustrate the timeline view of task placements and memory use in Figure 7 . The memory use is shown with number of tiles as well as bytes over time with and without memory restriction. The tasks are numbered with its task IDs. The bigger tasks represent conv task while the smaller tasks represents pooling tasks. The time between tasks placements is the

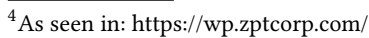

${ }^{5}$ We choose a slightly higher depth dimension of 16 compared to 6 in the original.

${ }^{6}$ Having a more balanced dimension in $\mathrm{X}, \mathrm{Y}$ and $\mathrm{Z}$.
}
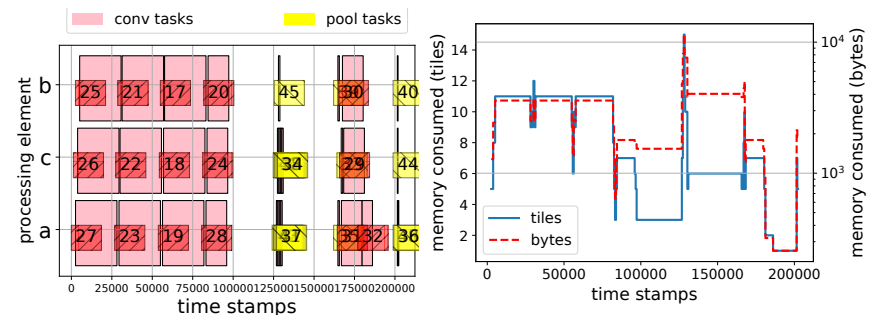

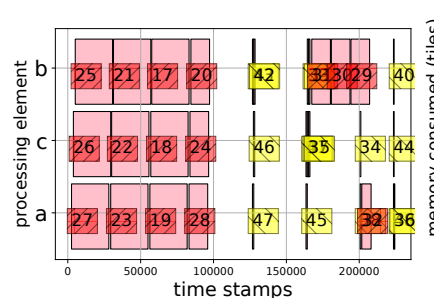

(a) Schedule of tasks

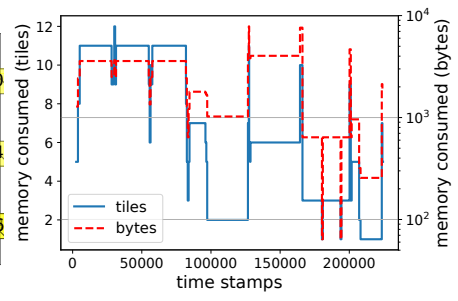

(b) Memory usage profile
Figure 7: DAG (Input $8 \times 8 \times 64$ and conv $8 \times 8 \times 64$ pooling $4 \times$ $256 \times 4)$ without (top) and with (bottom) memory restriction

tile load costs. We observe that a task based representation can interleave tasks across layers, thus, blurring the lines of layer-by-layer processing. We show that with memory restriction (bottom half of Figure 7) this DAG trade-offs better memory use (20\%) reduction at the expense of a slight increase in makespan (10\%) increase.

Finally, we illustrate the benefits of this task based scheduling over conventional layer-by-layer processing. The memory requirement in the latter for the input-conv boundary of the chosen LeNet5 requires $13568 \mathrm{~B}$ of memory ${ }^{7}$ and for the conv-pool boundary requires $15680 \mathrm{~B}$ of memory. We show the actual memory used (in bytes) for our task based approach in Figure 7. Taking the maximum memory used in both cases, we require $23.4 \%$ less memory (approximately 12000B).

\section{CONCLUSIONS}

There is an emerging opportunity to design accelerators to support on-device processing of deep neural network inferences. However, the research community lacks tools to understand the impact of software implementation choices on its design and vice versa.

We propose to model the dataflow in a deep convolutional neural networks (ConvNet) as a task-dependency graph to explore the ConvNet as a classical task-parallel mapping problem for a base accelerator system. We demonstrate the potential of such a representation using the first three layers of the LeNet-5 ConvNet in a constrained shared memory environment by developing a memoryaware heuristic for the original Heterogeneous Earliest Finish Time (HEFT) scheduling strategy. We explore various tiling and mapping configurations of task graphs under memory-constraint aware HEFT and compare it to the original HEFT.

Our results on the first three layers of LeNet-5 shows that DAGs with tiles having a large dimension along one axis does not perform better in makespan and memory compared to DAGs with regular shaped tiles. Decreasing the shared memory size results in some

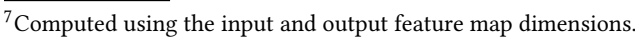


DAGs that trade-off memory use at the expense of makespan. Finally, we highlight that task-based approach offers better memory use by exploiting cross-layer dependencies compared to conventional approaches of layer-by-layer processing.

Future work, would be to study the impact of this DAG representation on other architectural features such as number of processing elements, heterogeneous processors and others. We also aim to apply a task-based approach to ConvNets with layers having residual connections and depthwise convolutions, such as those found in ResNet and MobileNet.

\section{ACKNOWLEDGMENTS}

This research was conducted with support for C. Rodrigues and G.D. Riley from the IS-ENES2 project, funded under the European FP7INFRASTRUCTURES-2012-1 call (GA No: 312979). C. Rodrigues is also part-funded by Arm under a PhD Studentship Agreement. M. Luján is supported by a Royal Society University Research Fellowship. The author would also like to acknowledge the valuable insights and guidance of Richard Neill, Nuno Miguel Nobre and Samuele Vinanzi at The University of Manchester and Rune Holm at Arm, Cambridge.

\section{REFERENCES}

[1] Manoj Alwani, Han Chen, Michael Ferdman, and Peter Milder. 2016. Fused-layer CNN accelerators. In The 49th Annual IEEE/ACM International Symposium on Microarchitecture. IEEE Press, 22.

[2] Leonardo Cecconi, Sander Smets, Luca Benini, and Marian Verhelst. 2017. Optimal tiling strategy for memory bandwidth reduction for cnns. In International Conference on Advanced Concepts for Intelligent Vision Systems. Springer, 89-100.

[3] Yu-Hsin Chen, Joel Emer, and Vivienne Sze. 2016. Eyeriss: A spatial architecture for energy-efficient dataflow for convolutional neural networks. In $A C M$ SIGARCH Computer Architecture News, Vol. 44. IEEE Press, 367-379.

[4] Mingyu Gao, Jing Pu, Xuan Yang, Mark Horowitz, and Christos Kozyrakis. 2017 Tetris: Scalable and efficient neural network acceleration with 3d memory. ACM SIGOPS Operating Systems Review 51, 2 (2017), 751-764.

[5] Kaiming He, Xiangyu Zhang, Shaoqing Ren, and Jian Sun. 2015. Deep Residual Learning for Image Recognition. arXiv preprint arXiv:1512.03385 (2015).

[6] Julien Herrmann, Loris Marchal, and Yves Robert. 2014. Memory-aware list scheduling for hybrid platforms. In Parallel \& Distributed Processing Symposium Workshops (IPDPSW), 2014 IEEE International. IEEE, 689-698.

[7] Andrew G Howard, Menglong Zhu, Bo Chen, Dmitry Kalenichenko, Weijun Wang, Tobias Weyand, Marco Andreetto, and Hartwig Adam. 2017. Mobilenets Efficient convolutional neural networks for mobile vision applications. arXiv preprint arXiv:1704.04861 (2017).

[8] Norman P Jouppi, Cliff Young, Nishant Patil, David Patterson, Gaurav Agrawal, Raminder Bajwa, Sarah Bates, Suresh Bhatia, Nan Boden, Al Borchers, et al. 2017. In-datacenter performance analysis of a tensor processing unit. In Proceedings of the 44th Annual International Symposium on Computer Architecture. ACM, 1-12.

[9] Alex Krizhevsky, Ilya Sutskever, and Geoffrey E. Hinton. 2012. ImageNet Classification with Deep Convolutional Neural Networks. (2012), 1097-1105.

[10] Nicholas D Lane, Sourav Bhattacharya, Petko Georgiev, Claudio Forlivesi, and Fahim Kawsar. 2015. An Early Resource Characterization of Deep Learning on Wearables, Smartphones and Internet-of-Things Devices. In Proceedings of the 2015 International Workshop on Internet of Things towards Applications. ACM, 7-12.

[11] Yann Lecun, Leon Bottou, Yoshua Bengio, and Patrick Haffner. 1998. Gradientbased learning applied to document recognition. In Proceedings of the IEEE.

[12] Ananda Samajdar, Yuhao Zhu, Paul Whatmough, Matthew Mattina, and Tushar Krishna. 2018. SCALE-Sim: Systolic CNN Accelerator. arXiv preprint arXiv:1811.02883 (2018)

[13] Karen Simonyan and Andrew Zisserman. 2014. Very deep convolutional networks for large-scale image recognition. arXiv preprint arXiv:1409.1556 (2014).

[14] Arthur Stoutchinin, Francesco Conti, and Luca Benini. 2019. Optimally Scheduling CNN Convolutions for Efficient Memory Access. arXiv preprint arXiv:1902.01492 (2019).

[15] Haluk Topcuoglu, Salim Hariri, and Min-you Wu. 2002. Performance-effective and low-complexity task scheduling for heterogeneous computing. IEEE transactions on parallel and distributed systems 13, 3 (2002), 260-274.
[16] Xuan Yang, Jing Pu, Blaine Burton Rister, Nikhil Bhagdikar, Stephen Richardson, Shahar Kvatinsky, Jonathan Ragan-Kelley, Ardavan Pedram, and Mark Horowitz. 2016. A systematic approach to blocking convolutional neural networks. arXiv preprint arXiv:1606.04209 (2016) 